\title{
A Two-Material Miniature Specimen Test Method and the Associated Inverse Approach for High Temperature Applications
}

\author{
W. Wen ${ }^{1, *}$, W. Sun ${ }^{1}$ and A.A. Becker ${ }^{1}$ \\ ${ }^{1}$ Faculty of Engineering, University of Nottingham, University Park, Nottingham NG7 2RD, UK \\ *Correspondence: Mr. Wu Wen \\ E-mail: wenwu.gm2011@gmail.com; Tel.: 07526973730; Address: Room C03 ITRC Building, University Park \\ Nottingham, University Park, Nottingham, UK NG7 2RD
}

\begin{abstract}
A miniature two-material test specimen has been developed for material property characterization. Elasticplastic and creep damage models have been applied for a semi-analytical model and a finite element (FE) model to simulate the miniature specimen tensile and creep tests on a two-material system. An inverse optimization algorithm has been developed, which can be used to extract the creep properties of the unknown material in the two-material system. Single material miniature specimen tensile tests have been performed for an aluminum alloy at room temperature and $400^{\circ} \mathrm{C}$ and full stage creep tests have been performed for a P91 steel at $650^{\circ} \mathrm{C}$. All the tensile and creep test specimens are brought to fracture failure. The experimental results have been compared to the results from the corresponding results from conventional uniaxial tensile and creep tests on the same materials and under the same test conditions. This miniature specimen testing technique and the developed inverse method have the potential to become a new approach for determining the elastic-plastic and the full-stage creep properties until rupture for a two-material system at high temperature, e.g. a coating-substrate system.
\end{abstract}

Keywords: Miniature Specimen; Two-Material; Creep Damage; Creep Fracture; Finite Element; Inverse Method

\section{Nomenclature}

\begin{tabular}{lll}
\hline Symbol & Definition & Units \\
\hline $\boldsymbol{h}_{\mathbf{1}}, \boldsymbol{h}_{\mathbf{2}}$ & Thicknesses & $\mathrm{mm}$ \\
$L, \boldsymbol{L}_{\mathbf{0}}$ & Lengths & $\mathrm{mm}$ \\
$P$ & Force & $\mathrm{N}$ \\
$\boldsymbol{S}_{\mathbf{1}}, \boldsymbol{S}_{\mathbf{2}}$ & Cross-sectional areas & $\mathrm{mm}^{2}$ \\
$\boldsymbol{t}$ & time & $\mathrm{h}$ \\
$\boldsymbol{\sigma}, \boldsymbol{\sigma}_{\mathbf{1}}, \boldsymbol{\sigma}_{2}, \boldsymbol{\sigma}_{\boldsymbol{e}}, \boldsymbol{\sigma}_{\boldsymbol{r}}, \boldsymbol{\sigma}_{\mathfrak{j}}$ Stresses & $\mathrm{MPa}$ \\
$\boldsymbol{\sigma}_{\boldsymbol{y} \mathbf{1}}, \boldsymbol{\sigma}_{\boldsymbol{y} \mathbf{2}}$ & & \\
$\boldsymbol{u}, \boldsymbol{u}_{\mathbf{1}}, \boldsymbol{u}_{\mathbf{2}}$ & Displacements & $\mathrm{mm}$ \\
$\boldsymbol{\varepsilon}, \boldsymbol{\varepsilon}_{\mathbf{1}}, \boldsymbol{\varepsilon}_{\mathbf{2}}$ & Strains & \\
$\dot{\boldsymbol{\varepsilon}}^{\boldsymbol{c}}$ & Creep strain rate & $\mathrm{h}$ \\
$\boldsymbol{E}, \boldsymbol{E}_{\mathbf{1}}, \boldsymbol{E}_{\mathbf{2}}$ & Young's moduli & $\mathrm{GPa}$ \\
$\boldsymbol{A}, \boldsymbol{B}, \boldsymbol{n}, \chi, \boldsymbol{\phi}$ & Creep properties & \\
$\boldsymbol{\omega}, \boldsymbol{\omega}_{\mathbf{1}}, \boldsymbol{\omega}_{\mathbf{2}}$ & Creep damage parameters & \\
$\boldsymbol{\beta}, \boldsymbol{\eta}$ & Reference stress conversion factors & \\
\hline
\end{tabular}

\section{Introduction}

A high temperature environment is a common operating condition for many engineering applications, such as power plants, chemical plants, gas turbines and aero-engines. Failure of high temperature components occurs often due to creep 
fracture and creep damage. In many cases, it is desirable to assess the material behavior and predict the future performance of certain components or structures for economic efficiency and risk management. For instance, creep strain and damage, which occur at elevated temperatures for extended periods of time could lead to catastrophic failures of critical components or structures. A coating-substrate system is a typical case in the scenario of advanced high temperature applications. Usually the thickness of the coating is small and it is difficult to extract a large specimen for a conventional uniaxial creep test. Therefore, a miniature specimen testing technique is particularly required in this case. Various types of small specimen testing technique have been used for mechanical and creep property characterization [1, 2]. These include the impression creep test (ICT) (e.g. [3, 4]), small punch creep test (SPCT) (e.g. [5-7]), small ring creep test (SRCT) (e.g. [8, 9]) and small two-bar creep test (STBCT) (e.g. [10, 11]). Figure 1 shows a schematic diagram of the small specimen configurations and loading arrangements. The ICT and the SRCT are suitable for determining minimum creep strain rate data but are not able to provide the tertiary stage creep data. The STBCT provides full stage creep data, but it is difficult to determine which bar fails first. The SPCT can also provide full stage creep data, but the data is difficult to interpret due to the complex nature of the physical deformation mechanism. The most challenging issue with the SPCT is the conversion or relationship of the experimental data to the uniaxial creep test. A code of practice was developed to convert the load and displacement data from SPCT to stress and strain data in 2006 [12]. Although this code of practice has been accepted by many researchers, the relationships are empirical and have been developed only for a specific testing geometry. It would be useful if an alternative small specimen testing method with a more straightforward relationship to the conventional creep test can be developed. There are studies aiming to extract the material properties from mechanical tests using miniature specimens geometrically similar to the conventional testing specimens (e.g. [13-16]). Generally, the agreements reported between the miniature and standard specimen tests are promising, though differences are observed despite that the sizes of some specimens used in the literature are relatively large.

The use of inverse method could provide a tool for improved data interpretation. Many inverse methods have been proposed to determine the elastic-plastic properties (e.g. [17-23]) from various of testing methods. However, inverse methods have not been commonly used for the characterization of creep properties (e.g. [7, 21]). The feasibility of extracting steady-state creep properties using an inverse method has been demonstrated, though the creep damage properties have not been included [7]. There are also studies on the creep behavior of two-material and multi-material systems (e.g. [24-28]). It has been shown that the creep damage properties can be determined numerically from the straintime curves obtained from the uniaxial creep tests data based on certain assumptions.

The objective of the current study is to develop a miniature specimen testing technique and an inverse method to determine the elastic-plastic and creep damage properties of an unknown material from a two-material system. The proposed testing method can be directly related to the conventional uniaxial test. Single material miniature specimen tensile tests and creep tests were carried out on an aluminum alloy and a P91 steel. Future tests on the miniature coatingsubstrate specimen are planned. The inverse method developed shows the potential to extract full stage creep damage properties of an unknown material from the compound creep testing results of a two-material system based on theoretical experiments of the two-material system.

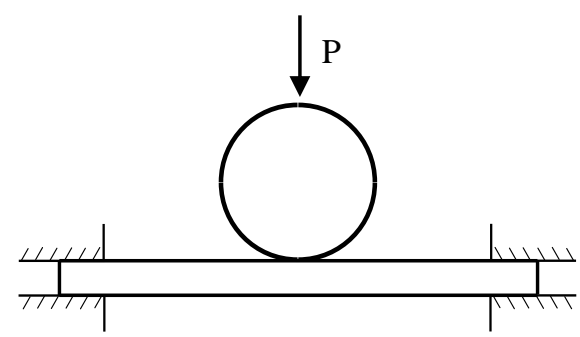

(a) SPCT

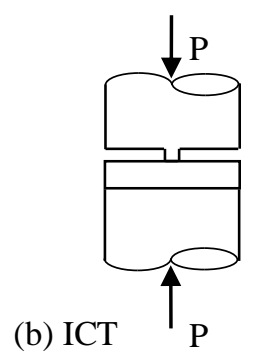

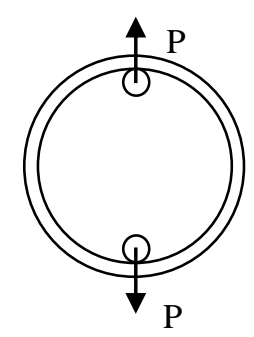

(c) SRCT

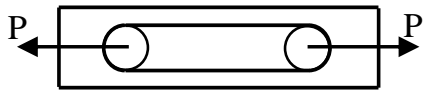

(d) STBCT

Figure 1. Schematic diagrams showing the small specimen types and loading arrangements: (a) SPCT; (b) ICT; (c) SRCT; and (d) STBCT. 


\section{Two-Material System}

The general methodology of the current study includes 1) carrying out single-material tests for a substrate material and a coating material to obtain the single material responses; 2) carrying out two-material system (e.g. coating on substrate) tests to obtain the compound responses; 3) applying the developed inverse method to determine the unknown material properties; 4) using the determined material properties for semi-analytical solutions or FE modelling of the single-material tests and comparing the predicted material behavior to the experimental results to validate the reliability and accuracy of the inverse method.

A two-material specimen in tension is shown in Figure 2. The two layers have equal length, $L$, equal width, $d$, and different thicknesses, $h_{1}$ and $h_{2}$, therefore different cross-sectional areas, $S_{1}$ and $S_{2}$. It is assumed that the properties of one of the materials are known in this study (e.g. the substrate material properties are known for a coated system). Since $h_{1}$ and $h_{2}$ are much smaller than $L$, it is assumed that the stresses in material 1 and 2 in the uniform section are in a uniaxial state, i.e. the axial strains in the uniform section are identical.

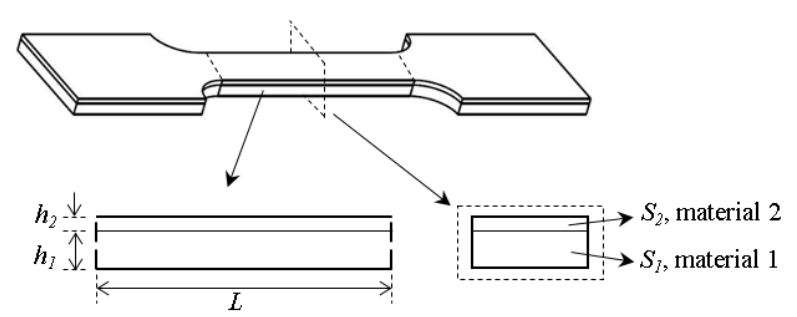

Figure 2. A two-material specimen in tension.

\subsection{Tensile Behavior}

The equilibrium equation for the two-material system under tensile force, $\mathrm{P}$, can be expressed as follows:

$$
\sigma_{1} S_{1}+\sigma_{2} S_{2}=P
$$

where $\sigma_{1}, \sigma_{2}$ are the stresses in materials 1 and 2, respectively. $S_{1}$ and $S_{2}$ are the cross-sectional areas of materials 1 and 2 , respectively. The compatibility equation (the two layers having equal displacements) is:

$$
u_{1}=u_{2}=u
$$

or

$$
\varepsilon_{1}=\varepsilon_{2}=\varepsilon
$$

where $u_{1}, u_{2}$ and $\varepsilon_{1}, \varepsilon_{2}$ are the displacements and strains in materials 1 and 2, respectively. Assuming a power law hardening behavior, the elastoplastic stress-strain relationships are expressed as follows:

$$
\sigma=\left\{\begin{array}{c}
\mathrm{E} \varepsilon, \varepsilon<\frac{\sigma_{y}}{E} \\
\mathrm{~K}^{n}, \varepsilon \geq \frac{\sigma_{y}}{E}
\end{array}\right.
$$

where $\mathrm{K}=E^{n}{\sigma_{y}}^{1-n}$. E, $\sigma_{y}$ and $n$ are the Young's modulus, yield stress and hardening exponent, respectively. The stress in the known material 1 can be determined as follows:

$$
\sigma_{1}=\left\{\begin{aligned}
E_{1} \varepsilon, & \varepsilon<\frac{\sigma_{y 1}}{E_{1}} \\
E_{1}{ }^{{ }^{1}} \sigma_{y 1}{ }^{1-n_{1}} \varepsilon^{n_{1},} \varepsilon & \geq \frac{\sigma_{y 1}}{E_{1}}
\end{aligned}\right.
$$

where $E_{1}, \sigma_{y 1}$ and $n_{1}$ are the Young's modulus, yield stress and hardening exponent of material 1, respectively. The stress in the unknown material 2 can be determined from Eqs (1) and (3): 


$$
\sigma_{2}=\frac{P-\sigma_{1} S_{1}}{S_{2}}
$$

The constitutive equations for material 2 are as follows:

$$
\sigma_{2}=\left\{\begin{aligned}
E_{2} \varepsilon, & \varepsilon<\frac{\sigma_{y 2}}{E_{2}} \\
E_{2}{ }^{{ }^{n}} \sigma_{y 2}{ }^{1-n_{2}} \varepsilon^{n_{2}}, & \varepsilon \geq \frac{\sigma_{y 2}}{E_{2}}
\end{aligned}\right.
$$

where $E_{2}, \sigma_{y 2}$ and $n_{2}$ are the Young's modulus, yield stress and hardening exponent of material 2, respectively. These material constants can be determined numerically.

\subsection{Creep Behavior}

\subsubsection{Steady-State Creep}

For steady-state creep, the creep strain rate, $\dot{\varepsilon}^{c}$, can be expressed as follows:

$$
\dot{\varepsilon}^{c}=A \sigma^{n}
$$

where $\sigma$ is the stress and $A$ and $n$ are material constants. Assuming that the properties of material 1 , i.e. $A_{1}$ and $n_{1}$, are known, the stress in material 1 can be determined with the measured strain rate $\dot{\varepsilon}^{c}$ using Eq (8) as follows:

$$
\sigma_{1}=\left(\frac{\dot{\varepsilon}^{c}}{A_{1}}\right)^{\frac{1}{n_{1}}}
$$

with the equilibrium and compatibility equations (1)-(3), the stress in material 2 can be determined as follows:

$$
\sigma_{2}=\frac{P-\left(\frac{\dot{\varepsilon}^{c}}{A_{1}}\right)^{\frac{1}{n_{1}}} S_{1}}{S_{2}}
$$

The creep properties of material 2, $A_{2}$ and $n_{2}$, can be determined numerically from the following constitutive equation:

$$
\dot{\varepsilon}^{c}=A_{2} \sigma_{2}{ }_{2}=A_{2}\left(\frac{P-\left(\frac{\dot{\varepsilon}^{c}}{A_{1}}\right)^{\frac{1}{n_{1}}} S_{1}}{S_{2}}\right)^{n_{2}}
$$

\subsubsection{Creep Damage}

A Kachanov type creep damage model can be used to represent the creep behavior (e.g. [29]). The formulations for the case of uniaxial stress state can be expressed as follows:

$$
\begin{aligned}
& \dot{\varepsilon}^{c}=A\left(\frac{\sigma_{e q}}{1-\omega}\right)^{n} \\
& \dot{\omega}=B \frac{\sigma_{r}^{\chi}}{(1-\omega)^{\phi}}
\end{aligned}
$$

where $\sigma_{\mathrm{eq}}$ and $\sigma_{\mathrm{r}}$ are the equivalent and rupture stresses, respectively $\left(\sigma_{\mathrm{eq}}=\sigma_{\mathrm{r}}\right.$ in the case of uniaxial stress $) ; \omega(0 \leq \omega$ $\leq 1$ ) is a damage variable; $A, n, B, \chi$, and $\phi$ are material constants. For material 1 shown in Figure 1 , the constitutive equations are:

$$
\begin{aligned}
& \dot{\varepsilon}^{c}=A_{1}\left(\frac{\sigma_{1}}{1-\omega_{1}}\right)^{n_{1}} \\
& \dot{\omega}_{1}=B_{1} \frac{\sigma_{1} \chi_{1}}{\left(1-\omega_{1}\right)^{\phi_{1}}}
\end{aligned}
$$

Assuming the creep properties of material $1\left(A_{1}, n_{1}, B_{1}, \chi_{1}\right.$, and $\left.\phi_{1}\right)$ are known, the stress $\sigma_{1}$ can be determined from Eq (13b) by applying the experimentally determined minimum strain rate (MSR), $\dot{\varepsilon}^{c}{ }_{\text {min }}$, for the steady-state creep, during which there is no creep damage, i.e. $\omega_{1}=0$ : 


$$
\sigma_{1}=\left(\frac{\dot{\varepsilon}^{c} \min }{A_{1}}\right)^{\frac{1}{n_{1}}}
$$

With the equilibrium and compatibility equations (1)-(3) and Eq (14), the stress in material 2 can be determined as follows:

$$
\sigma_{2}=\frac{P-\sigma_{1} S_{1}}{S_{2}}=\frac{P-\left(\frac{\dot{\varepsilon}^{c} \min }{A_{1}}\right)^{\frac{1}{n_{1}}} S_{1}}{S_{2}}
$$

The creep constitutive equations of material 2 are as follows:

$$
\begin{aligned}
& \dot{\varepsilon}^{c}=A_{2}\left(\frac{P-\left(\frac{\dot{\varepsilon}^{c} \min }{A_{1}}\right)^{\frac{1}{n_{1}}} S_{1}}{S_{2}\left(1-\omega_{2}\right)}\right)^{n_{2}} \\
& \dot{\omega}_{2}=\frac{B_{2}}{\left(1-\omega_{2}\right)^{\phi_{2}}}\left(\frac{P-\left(\frac{\dot{\varepsilon}^{c} \min }{A_{1}}\right)^{\frac{1}{n_{1}}} S_{1}}{S_{2}}\right)^{\chi_{2}}
\end{aligned}
$$

where $A_{2}, n_{2}, B_{2}, \chi_{2}$, and $\phi_{2}$ are the unknown creep constants of material 2 , which can be determined numerically using Eq (15), (16a) and (16b).

\subsection{Forward Analysis of the Two-Material System}

Forward analysis can be performed for any two-material system with the known material properties based on the analysis presented in section 2.2. A demonstration of the forward analysis of a two-material creeping system with fictional material properties is presented in this section. The elastic-plastic behaviors were neglected for simplification since the elasticplastic deformation was relatively small in these cases. Table 1 shows the creep damage properties of two fictional materials M1 and M2. The forward analysis was implemented through a semi-analytical computing script developed based on the Kachanov creep damage model as presented in section 2.2.2. Since the creep properties of the two materials are known, the compatibility condition (equal creep strain rate), the equilibrium condition and the Kachanov creep damage equations can be used for semi-analytical solutions. To verify the accuracy of the analytical solutions, FE analysis was carried under the same conditions and with the same material properties shown in Table 1, using the commercial code Abaqus 6.14-1. A three-dimensional eight-node brick element with reduced integration (C3D8R) was used for the FE model. Figure 3 shows the stress contour obtained from the FE analysis (a quarter section of the specimen shown in Figure 2). The stresses in material 1 and 2 in most regions of the uniform section are close to be in uniaxial state, which is consistent with the assumption taken for the analytical solutions.

The comparison between the semi-analytical solutions and the FE results is shown in Figure 4. Figure 4(a) shows that the compound creep strains (the creep strains in the two material layers M1 and M2 are equal in both cases) obtained from semi-analytical solutions and FE analysis agree very well. Figure 4(b) shows the creep damage evolutions of the two materials. The damage history of the two material layers are different: the MSR in material M1 is much higher than that in material M2. The analytical solutions and the FE analysis show very close results again. For the semi-analytical solutions, the instantaneous stresses in the two materials were obtained semi-analytically using the instantaneous damage values and creep strain rates obtained from the Kachanov creep damage model. The slight differences found between the results from the semi-analytical solutions and FE analysis might be mainly due to that a uniaxial stress case was assumed for the analytical solutions while that was not exactly the case for the FE analysis (the stresses in some regions of the uniform section are not in the uniaxial state as shown in Figure 3). As a result, the equivalent stresses obtained from the FE analysis were slightly lower than the analytically obtained stresses. This explains the fact that the creep strains and creep damage values from the FE analysis are slightly lower than the corresponding analytical results, as shown in Figures 4(a) and (b).

Table 1. The material properties of the fictional materials M1 and M2.

\begin{tabular}{cccccccc}
\hline \hline Material & $E$ & $\boldsymbol{\nu}$ & $A$ & $n$ & $B$ & $\chi$ & $\phi$ \\
\hline M1 & $150 \mathrm{GPa}$ & 0.3 & $5 \times 10^{-24}$ & 11 & $2 \times 10^{-22}$ & 11 & 5 \\
M2 & $180 \mathrm{GPa}$ & 0.3 & $5 \times 10^{-25}$ & 10 & $2 \times 10^{-24}$ & 10 & 4.5 \\
\hline
\end{tabular}




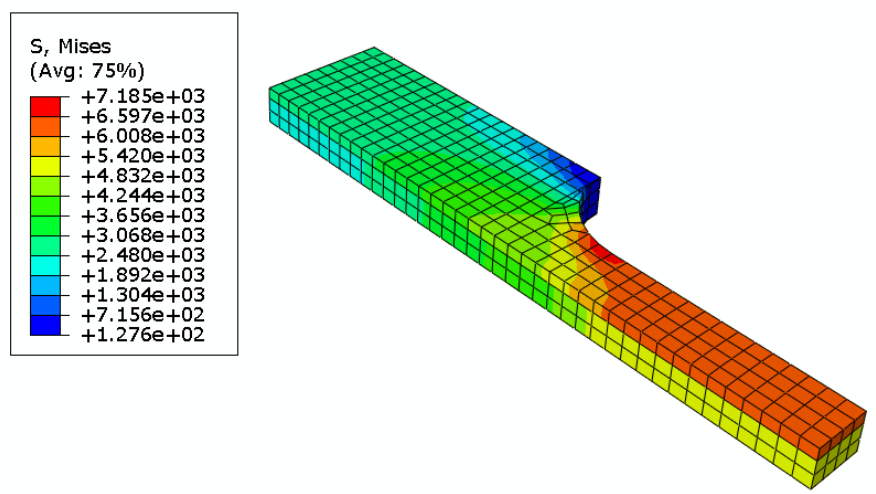

Figure 3. von Mises stress contour of the two-material system with the fictional materials M1 and M 2 obtained from FE analysis.
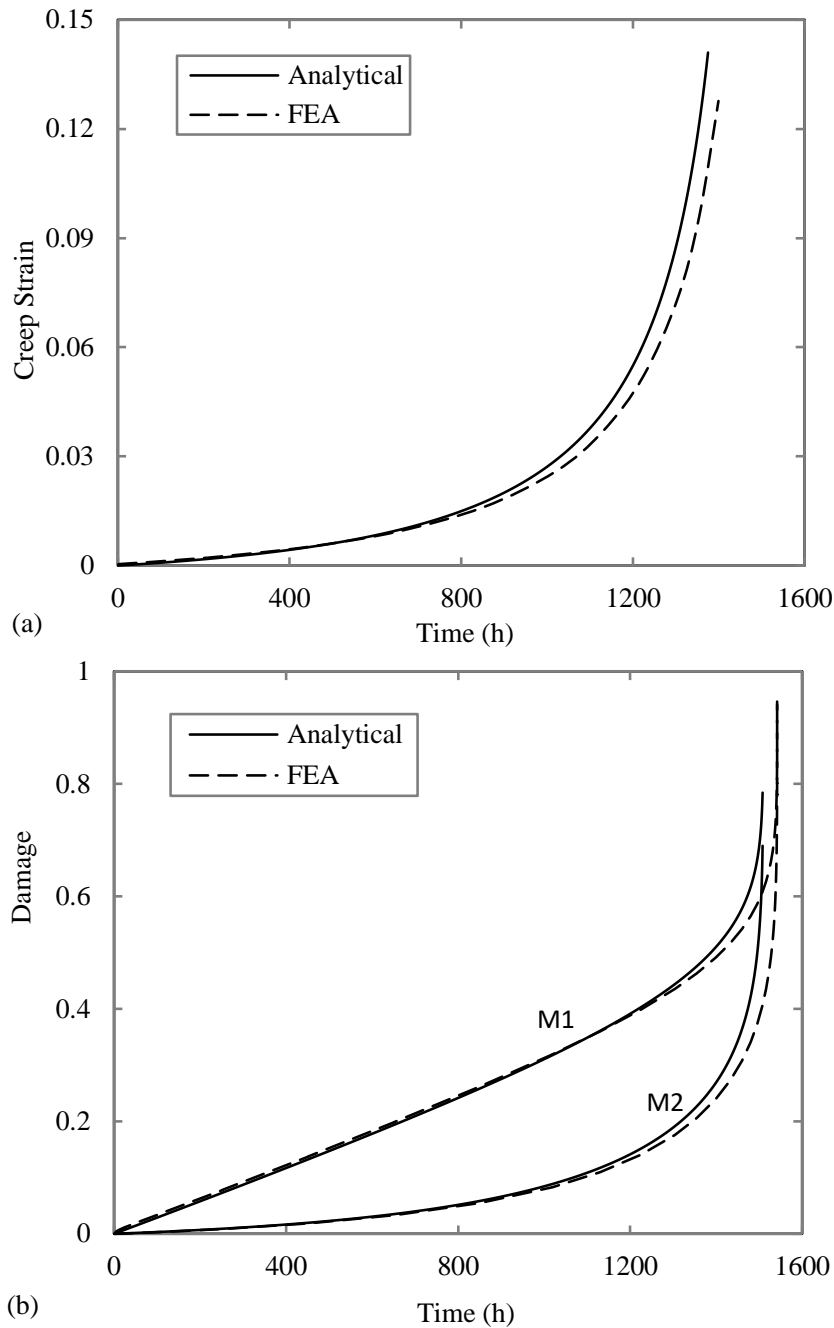

Figure 4. Forward creep analysis for the two-material system (M1 and M2) under the load of 42N: (a) creep strain (b) creep damage.

\section{Inverse Method and Application}




\subsection{The Inverse Method}

An inverse method was developed based on the forward analysis method presented in section 2.3. FE analysis can be avoided to save computing time since the results of the semi-analytical solutions have been proven to be very close to the FE analysis. Figure 5 shows a flowchart of the inverse algorithm. The experimental compound creep test results are supplied as the target values. The known material creep properties $\left(x_{1}\right)$ and an initial guess of the unknown material creep properties $\left(x_{2}\right)$ are supplied to the forward analysis method developed based on the Kachanov creep damage model (section 2.3). A nonlinear optimization algorithm is used to minimize the differences between the predicted compound creep responses and the experimental results until a set of optimized creep damage properties are obtained for the unknown material.

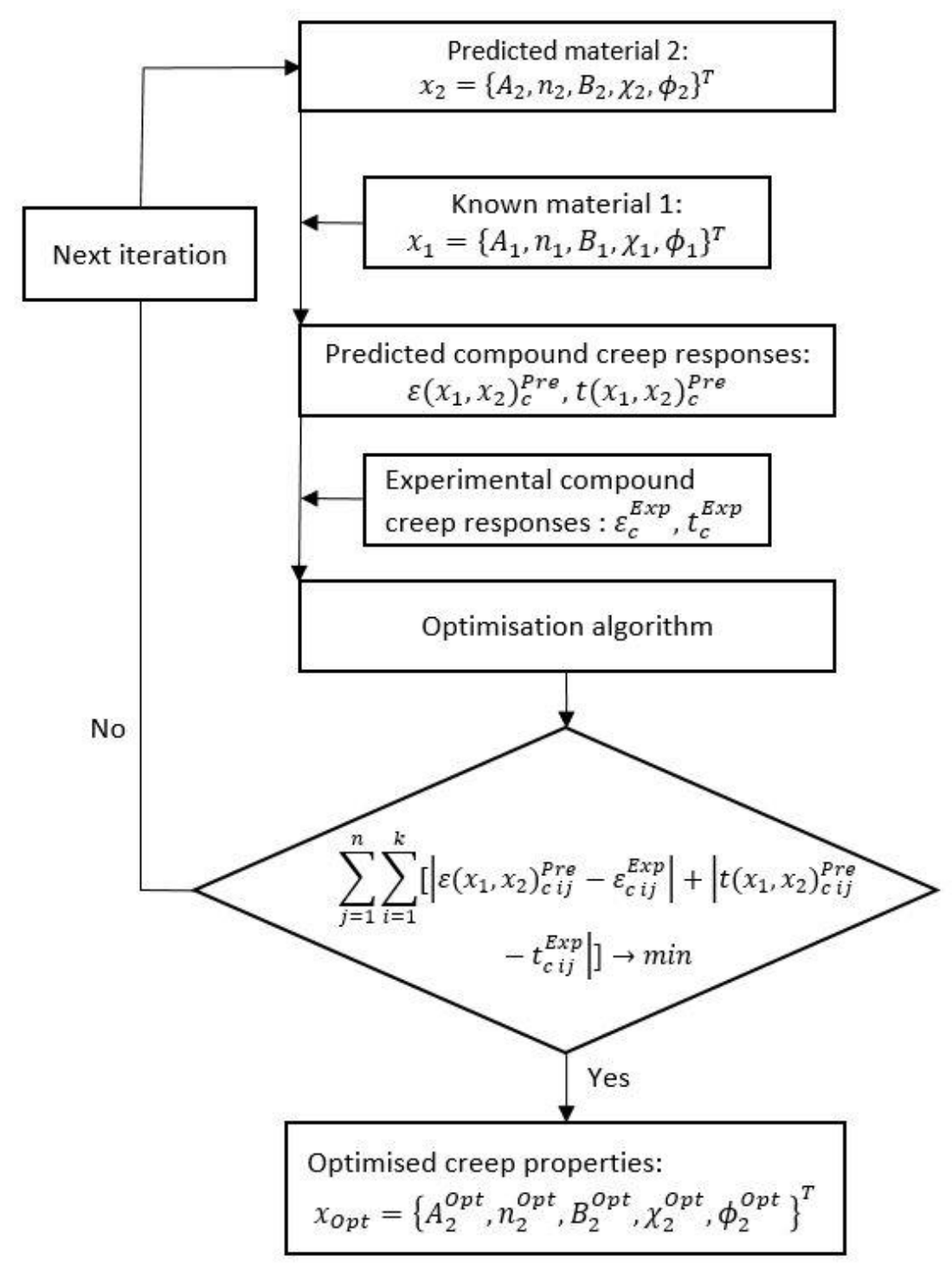

Figure 5. Flowchart of the inverse method.

\subsection{Demonstration of the Inverse Method}

To demonstrate the application of the proposed inverse method, it was applied to the two-material system used for the forward analysis presented in section 2.3, assuming that the properties for material M1 are known while the properties for material M2 are unknown. The objective was to determine the creep damage properties of M2 from the compound creep responses. Three different loads, $44 \mathrm{~N}, 46 \mathrm{~N}$ and $48 \mathrm{~N}$, were applied to the two-material system with the creep properties for materials M1 and M2 shown in Table 1 to produce three "theoretical" compound creep stress-strain curves (equivalent to the experimental compound creep responses shown in Figure 5) as the targets of the optimization. Figure 6 shows the strain-time curves obtained from the optimized creep damage properties compared to the theoretical experiment. The optimized results agree very well with the target curves for all three different loading forces regarding 
the creep strain rate and the time to failure. To evaluate the quality of the optimization, the creep strain-time curves were also computed using the creep properties of M2 and the optimized creep properties for a single-material model with three different loading forces: $75 \mathrm{~N}, 80 \mathrm{~N}$ and $85 \mathrm{~N}$. The comparison is shown in Figure 7. The agreement is still acceptable, i.e. the strains during the steady-state creep stage are very close for the material M2 (Table 1) and the optimized material in all three loading forces, although the deviation increases as the creep develops near to failure. These results reveal that the optimized creep properties are close to the actual properties of the target material M2. It should be noted that the current inverse optimization algorithm is still sensitive to the initial values of the unknown creep damage properties (e.g. some initial values could lead to difficulty in convergence to the desired tolerance or extended computing time). This sensitivity to the initial conditions should be minimized in further development.

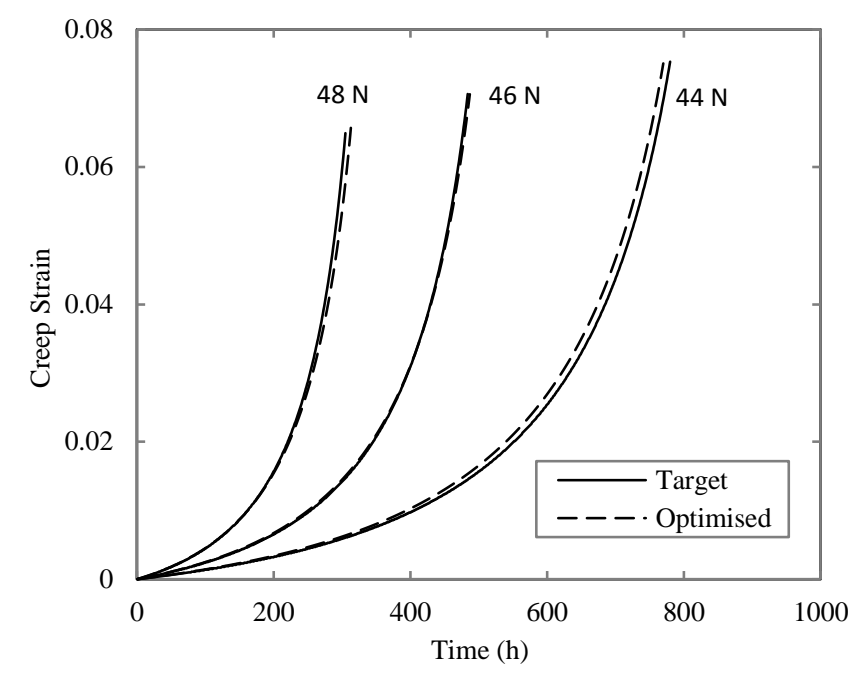

Figure 6. The inverse optimized curves and the target curves.

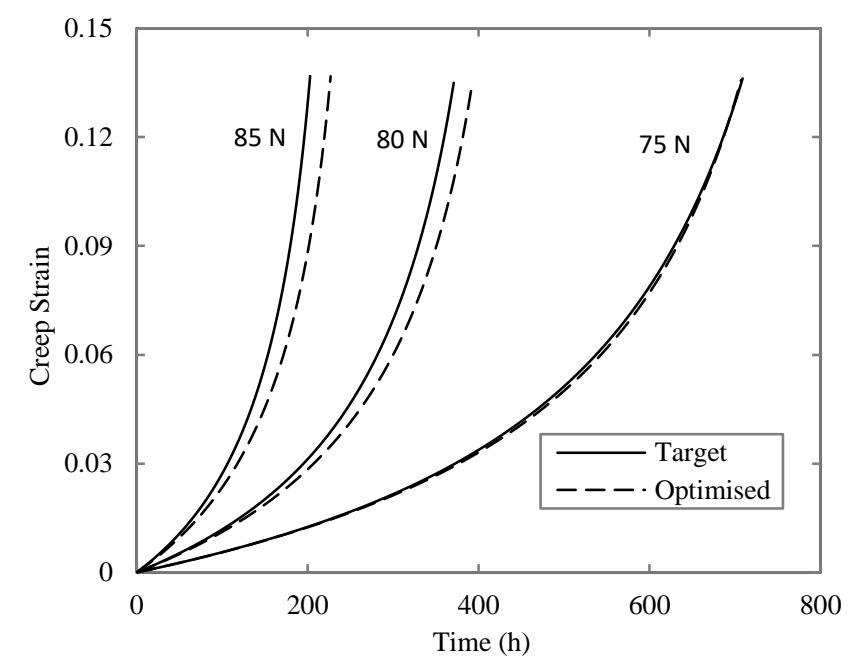

Figure 7. The single-material model FE analysis for material M2 (Table 1) and the optimized creep properties.

\section{Experimental Testing Using Single-Material Specimens}

A miniature specimen tensile/creep testing technique has been developed. Tensile tests have been carried out for an aluminum alloy with both miniature specimens and large specimens. Creep tests have been performed at $650^{\circ} \mathrm{C}$ on a P91 steel at different stress levels: $100 \mathrm{MPa}, 93 \mathrm{MPa}$ and $87 \mathrm{MPa}$ for both the miniature and large specimens. Figure 8 shows (a) the geometry and dimensions of the miniature specimen and (b) the loading assembly and experimental set-up with the specimen mounted to the machine (Tinius Olsen H5KS single column materials testing machine). The loading assembly were made of a high temperature resistant nickel-based superalloy, i.e. Nimonic 115. The specimen was clamped at both ends by specially manufactured clamps with machined surface features to strengthen the grip. The displacement was measured by two linear variable displacement transducer (LVDT) connected to both sides of the loading part of the machine. The mounted specimen was kept inside a furnace for the tests requiring heating. 


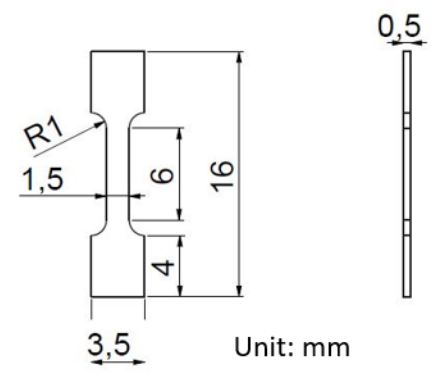

(a)

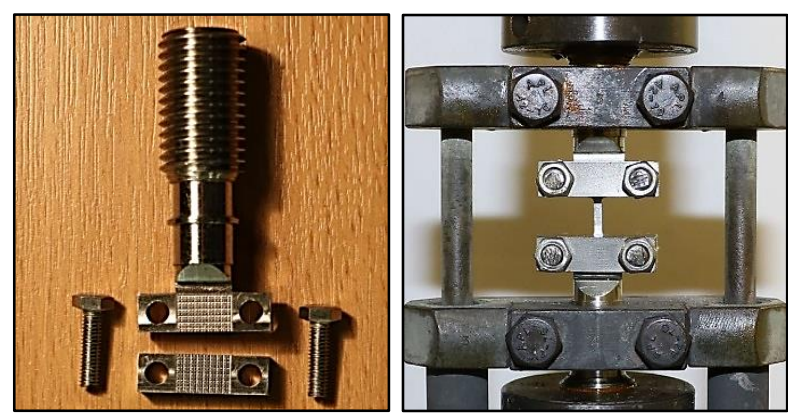

(b)

Figure 8. (a) Geometry and dimensions of the miniature specimen; (b) The loading assembly and experimental set-up.

\subsection{Miniature Specimen Tensile Tests on an Aluminum Alloy}

Tensile tests were carried out on an aluminum alloy to be compared to the tensile testing results obtained from the large specimen to validate the developed miniature testing method. The geometry of the large flat specimen was identical to that of the miniature specimen while the dimensions were six times of the miniature specimen, e.g. the thickness was $3 \mathrm{~mm}$ and the gauge length was $36 \mathrm{~mm}$. It is reported by Yuan et al [30] that the yield strength and ultimate tensile strength are independent of sample thickness for tensile tests on a rectangular cross-section specimen for thickness $>1 \mathrm{~mm}$.

Figure 9 shows the comparison between the tensile testing results obtained from the miniature specimens and the large specimens at different temperatures. The results from the two types of tests are generally comparable. It is noteworthy that the difference between the ultimate strengths obtained from the large and miniature specimens was much higher in percentage at $400^{\circ} \mathrm{C}$ than at RT. This could be partly due to the possible differences in the actual temperature of the specimens for the two types of tests (since the large specimen tests were carried out on a different machine) and its effect on the tensile properties of the material. For example, it is reported that the temperature effects on the tensile properties of a A319-Al alloy is minimum between -100 and $200^{\circ} \mathrm{C}$ while the yield strength, ultimate strength and the ductility varies drastically from 200 to $400^{\circ} \mathrm{C}$ [31], indicating that a small amount of temperature fluctuation could lead to a rather large difference in the tensile properties acquired from the tests at high temperatures. Also, the miniature specimen could be more sensitive to the accuracy of the manufacturing process, e.g. the possible small local variation in the thickness of the miniature specimen could lead to significant amount of difference in the loading force, hence the calculated stress, due to the miniaturized overall size. Similarly, possible surface oxidation might have more significant effects on the tensile responses of the miniature specimen due to the small thickness, though it needs further investigation for verification. 


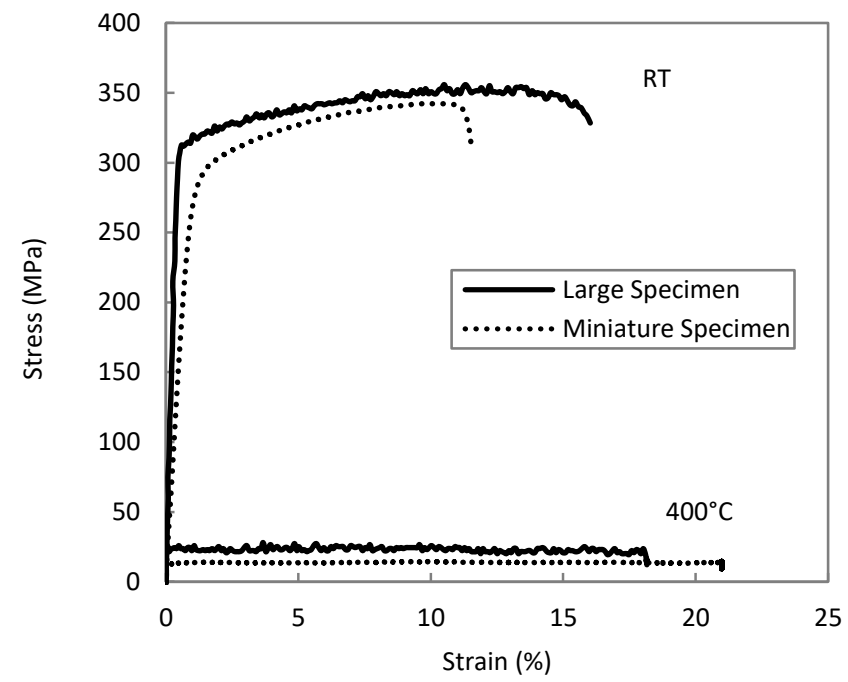

Figure 9. Miniature tensile test results on an aluminum alloy compared to the large specimen tensile test at different temperatures.

\subsection{Miniature Specimen Creep Test}

\subsubsection{Data Conversion}

It should be noted that the measurements were taken outside the gauge length region (at the clamped ends) for the miniature specimen tests because it is very difficult to fit a gauge into the very limited space. Therefore, the measured displacement was different from the actual displacement of the gauge length. To convert the data obtained from the miniature specimen creep test to the corresponding uniaxial data, a reference stress method was applied to determine the conversion relationships for stress and strain. The case of steady-state creep was applied for the demonstration.

Two conversion factors, $\eta$ and $\beta$, were introduced to define the conversion relationships as follows:

$$
\begin{aligned}
& \sigma_{\text {ref }}=\eta \sigma_{\text {nom }} \\
& \dot{\varepsilon}_{\text {ref }}=\frac{\dot{\Delta}}{\beta L_{0}}
\end{aligned}
$$

where $\sigma_{r e f}, \dot{\varepsilon}_{r e f}, \sigma_{n o m}, L_{0}$ and $\dot{\Delta}$ are the reference stress, reference strain rate, nominal stress, the uniform section length, and the measured displacement rate, respectively. $\beta L_{0}$ is defined as the equivalent gauge length. The conversion factors are determined by carrying out a series of FE analyses of the miniature specimen creep test. A Norton power law is assumed to simulate the creep behaviors of the material, i.e. the steady state creep strain rate $\dot{\varepsilon}^{c}$ is expressed as follows:

$$
\dot{\varepsilon}^{c}=A \sigma^{n}
$$

where $A$ and $n$ are material constants. The conversion factor $\beta$ can be expressed as follows:

$$
\beta=\frac{\dot{\Delta}}{A\left(\eta \sigma_{\text {nom }}\right)^{n} L_{0}}
$$

The conversion factors were assumed to depend solely on the specimen geometry and loading mode, so they should be material-independent. A series of FE analyses of the miniature specimen creep tests were carried out with different material constants $A$ and $n$. The parameter $n$, with the range of 2 to 12, was used for FE analysis. The corresponding value of $A$ for each $n$ value was calculated assuming a constant creep strain rate, i.e. $A_{0} \sigma^{n_{0}}=A_{i} \sigma^{n_{i}}$ for the convenience of FE analysis. The steady-state creep displacement-time curves obtained from the FE analyses are shown in Figure 10. It can be seen that the displacement rates, or strain rates, are very close to each other for different cases, indicating that the assumption of an approximately constant strain rate is valid. The minimum displacement rate, $\dot{\Delta}_{s s}$, was calculated for each curve. The objective was to find the value of $\eta$ with which the value of $\beta^{\prime}=\frac{\dot{\Delta}}{A\left(\alpha \sigma_{n o m}\right)^{n} L_{0}}$ becomes independent of $n$. Following the FE analysis, $\log \left(\beta^{\prime}\right)$ was calculated for a range of values of $\alpha(0.5$ to 1.2$)$ and the results are shown in 
Figure 11. It can be seen that $\log \left(\beta^{\prime}\right)$ is nearly constant for $\alpha=0.984(\alpha=\eta)$, independent of $n$. Therefore, the conversion factor $\beta$ can be calculated, i.e. $\beta=1.205$.

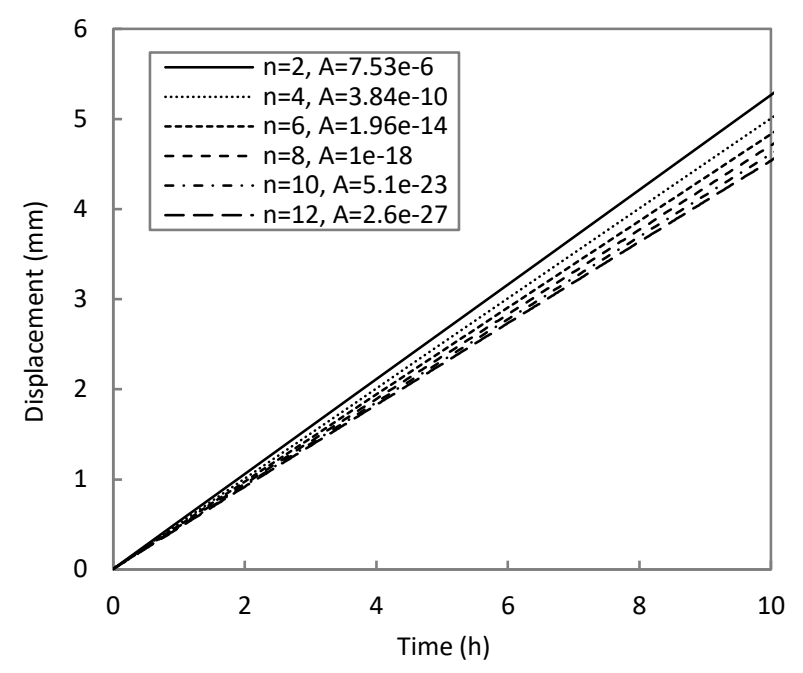

Figure 10. Steady-state creep displacement with variation of $n$ and $A$.

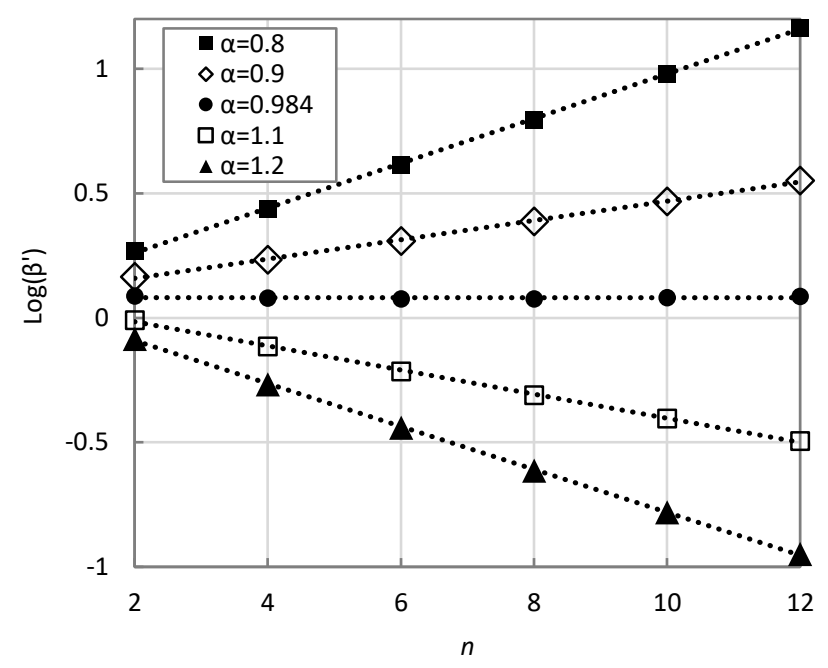

Figure 11. Determination of the conversion factors.

\subsubsection{Miniature Specimen Creep Testing Results of P91 Steel}

Preliminary creep tests were carried out on a P91 steel at $650^{\circ} \mathrm{C}$. The specimen geometry and the experimental set-up were the same as shown in Figure 6. Constant forces were applied to the specimen to achieve the stress levels of $100 \mathrm{MPa}, 93 \mathrm{MPa}$ and $87 \mathrm{MPa}$. Figure 12 shows the creep testing results (straintime curves) obtained from the miniature specimen and the corresponding large specimen uniaxial creep tests. The creep strain rates during the steady state agree well for the miniature specimen test and the uniaxial test at the stress level of $87 \mathrm{MPa}$. The miniature specimen tests show a higher strain rate and a shorter time to failure compared to the uniaxial tests at higher stress level such as $93 \mathrm{MPa}$ and $100 \mathrm{MPa}$. These differences might be due to the local stress concentration resulting from machining, e.g. near the clamp at the gauge length ends. Also, the testing results of the miniature specimen could be affected by the amount of clamping force applied to it, e.g. the pre-existing stress in the material before the creep test. In addition, the differences might be due to surface oxidation occurring during the tests. A photo of the fractured specimen from the miniature creep test of $87 \mathrm{MPa}$ is also shown in Figure 12. It shows a fracture surface of about $45^{\circ}$ angle and some degree of surface oxidation. The influences of surface oxidation might be amplified by the small thickness of the specimen. Figure 13 shows the variation of the MSR and the time to failure versus stress level for the uniaxial tests and the miniature tests, plotted in logarithmic scales. The approximate linear trends indicate the general consistency of 
the miniature specimen creep tests at different loading levels, although there are some differences between the uniaxial tests and the miniature tests.

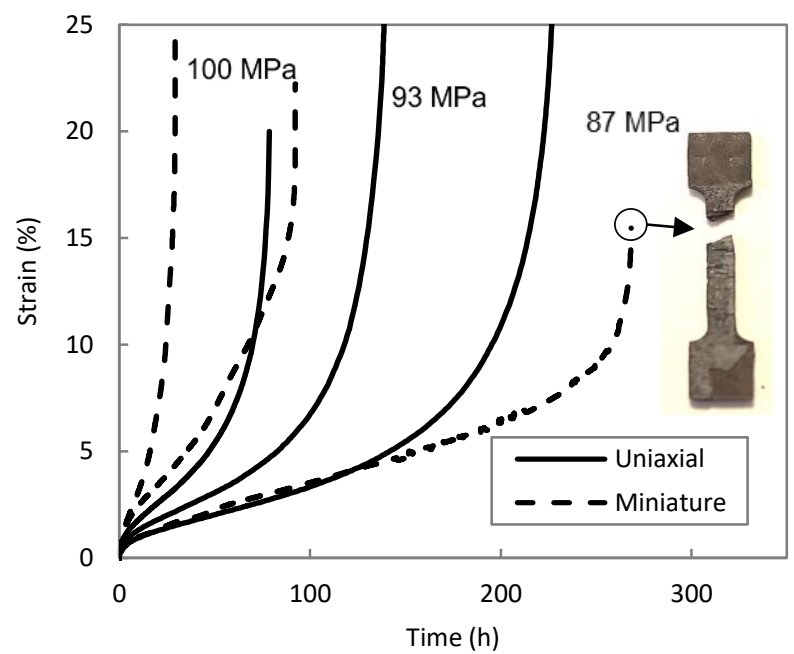

Figure 12. Miniature specimen and uniaxial creep tests on $\mathrm{P} 91$ steel at $650^{\circ} \mathrm{C}$.

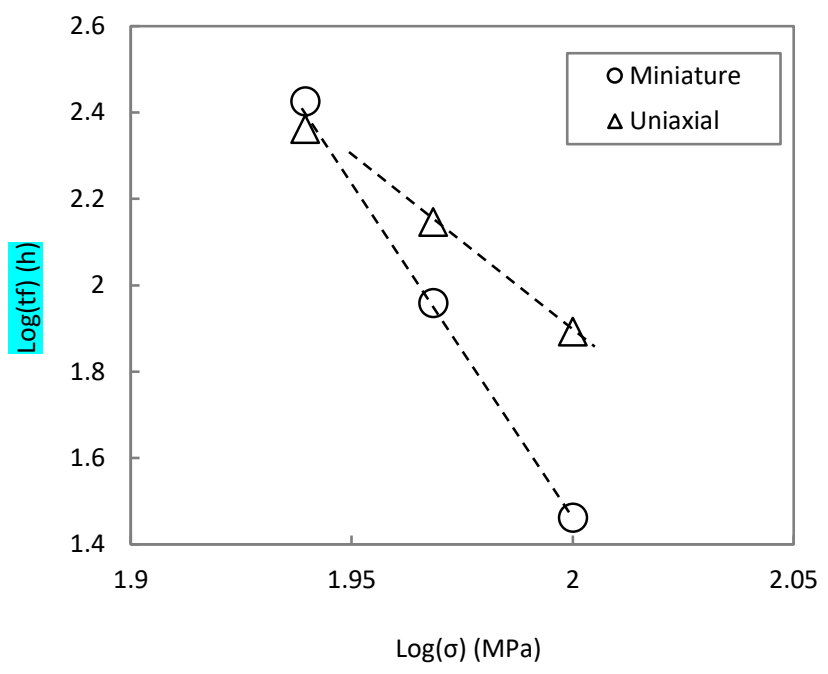

(a) 


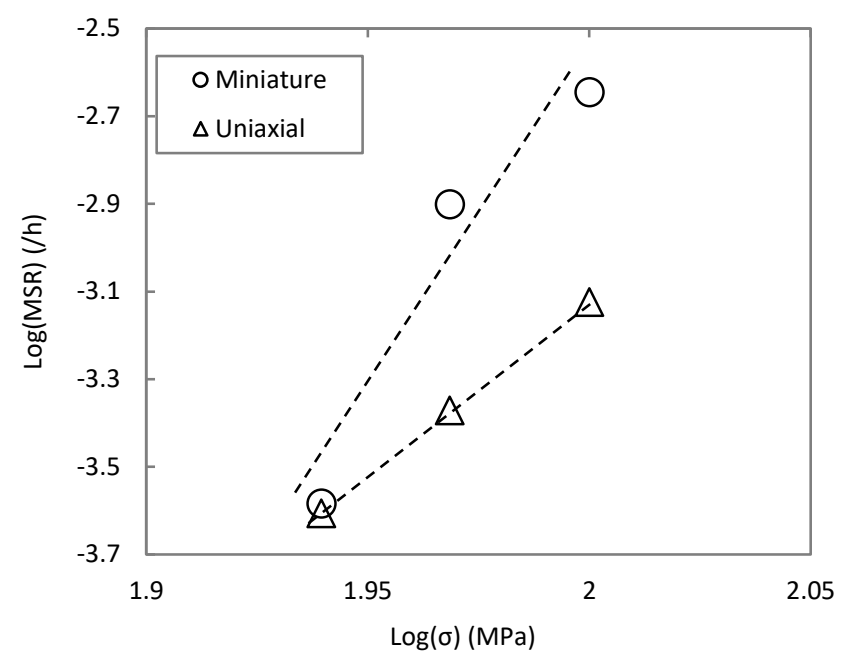

(b)

Figure 13. Variation of (a) the time to failure and (b) the MSR versus stress level, plotted on logarithmic scales: uniaxial creep tests and miniature creep tests.

\section{Conclusions}

The methodology development of a miniature specimen testing technique and the associated inverse method to determine the full-stage creep properties until fracture of an unknown material from the compound tensile and creep behaviors of a two-material system have been presented. The forward analysis and the inverse method have been demonstrated through a case study of a theoretical experiment. The semi-analytical solutions and the FE analysis showed very good agreement. The optimized compound creep behaviors obtained were very close to the target results. The differences might be due to that the actual stress distribution in the two material layers was not evaluated by the inverse optimization approach, since the stress was not a measurable quantity in the experiments. As a result, the stresses in the two material layers acted as the extra 'variables' during the optimization process.

Single-material miniature tensile tests and creep tests were carried out on an aluminum alloy and P91 steel, respectively. The results were compared to those from the large specimen uniaxial tests on the same materials. The results of the miniature specimen tests were generally comparable to the large specimen uniaxial tests while some differences were found. Those differences could be a result of the possible local stress concentration due to machining, the random errors in the manual handling procedures (e.g. the clamping locations), the preexisting strains and the surface oxidation. The testing technique might be improved by optimized specimen design for lower stress concentration factor, enhanced manufacturing quality, minimized influences from surface oxidation (e.g. carrying out the tests in a protective atmosphere or vacuum) and more accurate measurement and control of the testing temperature.

For future work, tensile and creep tests will be carried out on a coating-substrate system with the miniature specimen. The inverse method will be further improved regarding the efficiency and applied to the experimental results obtained from the extended single-material tests and coating-substrate miniature specimen tests. Full FE models will be used to simulate the realistic miniature specimen tensile/creep tests of the coated system.

Acknowledgments: This work was supported by the Engineering and Physical Sciences Research Council (EPSRC) through EPSRC Centre for Doctoral Training in Innovative Metal Process (IMPaCT, www.impact.ac.uk) [Grant Number EPL016206] and the project "Novel High Temperature Steam Transfer Pipes" [Grant number: EP-R000859-1]. The authors would also like to thank Mr. Shane Maskill for his assistance in the experimental work. 


\section{References}

[1] T.H. Hyde, W. Sun, J.A. Williams, The requirements for and the use of miniature test specimens to provide mechanical and creep properties of materials: - a review. Int. Mater. Rev. 52 (2007) 213-55.

[2] A. Morris, B. Cacciapuoti, W. Sun, The role of small specimen creep testing within a life assessment framework for high temperature power plant. Int. Mater. Rev. 63 (2018) 102-37.

[3] T.H. Hyde, W. Sun, A.A. Becker, Analysis of the impression creep test method using a rectangular indenter for determining the creep properties in welds, Int. J. Mech. Sci. 38 (1996) 1089-102.

[4] W. Sun, T.H. Hyde, S.J. Brett, Application of impression creep data in life assessment of power plant materials at high temperatures. J. Mats.: Design and Applications 222 (2008) 175-82.

[5] J. P. Rouse, F. Cortellino, W. Sun, T. H. Hyde, J. Shingledecker, Small punch creep testing: a review on modelling and data interpretation. Mater. Sci. Technol. 29 (2013) 1328-45.

[6] F. Cortellino, J. Rouse, B. Cacciapuoti, W. Sun, T. H. Hyde, Experimental and numerical analysis of initial plasticity in P91 steel small punch creep samples. Experimental Mechanics 57 (2017) 1193-212.

[7] Y.Z. Li, P. Stevens, J.F. Geng, D.F. Ma, L. Xu, Determination of creep properties from small punch test with reverse algorithm. Key. Eng. Mats. 734 (2017) 212-36.

[8] T. H. Hyde, W. Sun, A novel high sensitivity, small specimen creep test. J. of Strain Anal. Eng. 44 (2009) 171-85.

[9] W. Sun; T.H. Hyde, Determination of secondary creep properties using a small ring creep test technique. Metallurgical. J. 63 (2010) 185-93.

[10] W. Sun, T. H. Hyde, B. S. M. Ali, Small tensile bar specimen creep tests for use in determining creep deformation and rupture properties. 3nd Proc. Int. Conf. SSTT, ed. K. Matocha, R. Hurst, W. Sun. pp. 360-67. (C) OCELOT s.r.o. Ostrava, September 2014.

[11] A. Balhassn, T. H. Hyde, W. Sun, Analysis and design of a small, two-bar creep test specimen. Trans. ASME J. Eng. Mater. \& Technol. 135 (2013) 041006-1-041006-9.

[12] CEN CWA 15627 Workshop Agreement: Small punch test method for metallic materials (Part A), European Committee for Standardization, Brussels, December 2006.

[13] B. Yang, F. Xuan, J. Chen, Evaluation of the microstructure related strength of CrMoV weldment by using the in-situ tensile test of miniature specimen. Mater. Sci. Eng. A 736 (2018) 193-201.

[14] B. Yang, F. Xuan, Creep behavior of subzones in a CrMoV weldment characterized by the in-situ creep test with miniature specimens. Mater. Sci. Eng. A 723 (2018) 148-56.

[15] P. Dymácek, M. Jarý, F. Dobeš and L. Kloc, Tensile and creep testing of Sanicro 25 using miniature specimens. Materials 11 (2018) 142-52.

[16] K. Kumar, A. Pooleery, K. Madhusoodanan, R. N. Singh, J. K. Chakravartty, Use of miniature tensile specimen for measurement of mechanical properties. Procedia Engineering 86 (2014) 899-909.

[17] D. S. Schnur, N. Zabaras, An inverse method for determining elastic material properties and a material interface. Int. J. Num. Meth. Eng. 33 (1992) 2039-57.

[18] J. C. Gelin, O. Ghouati, An inverse method for determining viscoplastic properties of aluminium alloys. J. Mater. Proc. Tech. 45 (1994) 435-40.

[19] A. Husain, D. K. Sehgal, R. K. Pandey, An inverse finite element procedure for the determination of constitutive tensile behavior of materials using miniature specimen. Comp. Mater. Sci. 31 (2004) 84-92.

[20] J. J. Kang, A. A. Becker, W. Wen, W. Sun, Extracting elastic-plastic properties from experimental loading-unloading indentation curves using different optimization techniques. Int. J. Mech. Sci. 144 (2018) 102-09.

[21] O. Iracheta, C. J. Bennett, W. Sun, Characterisation of material property variation across an inertia friction welded CrMoV steel component using the inverse analysis of nano-indentation data. Int. J. Mech. Sci. 107 (2016) 253-63. 
[22] J. Kang, A. A. Becker, W. Sun, Determining elastic-plastic properties from indentation data obtained from finite element simulations and experimental results. Int. J. Mech. Sci. 62 (2012) 34-46.

[23] J. Lu; A. Campbell-Brown, S. Tu; W. Sun, Determination of creep damage properties from miniature thin beam bending using an inverse approach. Key Eng. Mats. 734 (2017) 260-72.

[24] T. H. Hyde, W. Sun, Determining creep properties for columnar and equiaxed regions of a $9 \mathrm{CrMoNbV}$ weld metal at $650^{\circ} \mathrm{C}$ using bulk uniaxial creep test data. Proc. of $3 \mathrm{rd}$ Int. Conf. on Integrity of High Temp Welds IoM Communications, London, April 2007, pp. 139-148.

[25] T. H. Hyde, W. Sun, A. Tang, P. J. Budden, An inductive procedure for determining the stresses in multi-material components under steady-state creep. J. of Strain Anal. Eng. 35 (2000) 347-58.

[26] T. H. Hyde, W. Sun, A. Tang, A general formulation of the steady-state creep deformation of multi-material components. Proc. of the 4th Int. Conf. on Modern Practice in Stress and Vibration Analysis, Nottingham, UK, Ed. A. A. Becker, September 2000, pp. 481-492.

[27] T. H. Hyde, K. Yehia, W. Sun, Observation on the creep of two-material structures. J. of Strain Anal. Eng. 31 (1996) 441-61.

[28] T. H. Hyde, W. Sun, Elastic-creep behavior of a three-material plate subjected to a bi-axial stress state. Mats. Sci. Eng. A 417 (2006) 174-81.

[29] L. Kachanov, On rupture time under condition of creep. Izvestia Akademi Naul USSR, Otd. Techn. Nauk, Moskwa, 1958, pp. 26-31.

[30] J. Yuan, Z. L. Zhang, Y. J. Su, L. J. Qiao, W. Y. Chu, Influence of specimen thickness with rectangular cross-section on the tensile properties of structural steels. Mater. Sci. Eng. A 532 (2012) 601-05.

[31] E. Rincón, H. F. López, M. M. Cisneros, H. Mancha, M. A. Cisneros, Effect of temperature on the tensile properties of an as-cast aluminum alloy A319. Mater. Sci. Eng. A 452-453 (2007) 682-87. 